\title{
Space weather and earthquakes: possible triggering of seismic activity by strong solar flares
}

\author{
Victor Novikov*,1, Yuri Ruzhin ${ }^{2}$, Valery Sorokin ${ }^{2}$, Alexey Yaschenko ${ }^{2}$ \\ (1) Joint Institute for High Temperatures of Russian Academy of Sciences, Moscow, Russian Federation \\ (2) Pushkov Institute of Terrestrial Magnetism, Ionosphere and Radio Wave Propagation of Russian Academy \\ of Sciences, Troitsk, Moscow region, Russian Federation
}

Article history: received October 12, 2018; accepted March 2, 2020

\begin{abstract}
The studies completed to-date on a relation of the Earth's seismicity and solar processes provided the fuzzy and contradictory results. The main problem of this research is a lack of physical explanation of a mechanism of earthquake triggering by strong variations of space weather conditions. Based on results obtained in the field and laboratory experiments on earthquake triggering by DC pulses injection into the Earth crust we may assume that the similar triggering phenomena may occur after the strong electromagnetic impact to the earthquake source due to solar flares or geomagnetic storms. Numerical estimations demonstrated that telluric currents induced by geomagnetic pulsations generated by solar flare have the similar density at the depth of earthquake source location $\left(10^{-6} \mathrm{~A} / \mathrm{m}^{2}\right)$ in comparison with the current density generated by artificial power sources $\left(10^{-7}-10^{-8} \mathrm{~A} / \mathrm{m}^{2}\right)$ resulted in observed spatiotemporal redistribution of seismic activity in the regions of Pamirs and Northern Tien Shan. For supporting the idea of a possible earthquake triggering by solar flares we carried out a statistical analysis of global and regional (Greece) seismicity behavior during the solar flare of X9.3 class occurred on September 6, 2017 (the strongest flare over the past thirteen years). We have discovered a new evidence of earthquake triggering due to the Sun-Earth interaction by simple comparison of a number of earthquakes before and after the strong solar flare. The global number of earthquakes (USGS catalog, $M \geq 4$ ) for time window of \pm 11 days after the solar flare has increased by $68 \%$, and the regional seismicity (Greece, EMSC catalog, $\mathrm{M} \geq 3$ ) has increased by $120 \%$.
\end{abstract}

Keywords: space weather, solar flare, telluric currents, earthquake triggering.

\section{Introduction}

It is well known that space weather, which incorporates a set of phenomena in the space including solar flares and magnetic storms, may affect technological systems and activities of modern civilization. The effects of geomagnetic disturbances on telegraph lines, submarine phone cables, and power systems have been noticed for over a century and described in a number of reviews [e.g., Lanzerotti and Gregori, 1986; Boteler et al., 1998]. In 


\section{Victor Novikov et al.}

addition, a discussion of an impact of strong variations of space weather on the lithosphere and possible triggering of earthquakes is under way. The problem of an influence of solar activity on the terrestrial processes is considered for more than 150 years starting from the study [Wolf, 1853] where it was pointed that sunspots may exert some influence on the earthquake occurrence. To-date quite a large number of studies have been completed to find a correlation between the Earth's seismicity and solar processes. Results of these statistical studies are contradictory: some researchers found the direct correlation between the energy or number of earthquakes and Wolf numbers [Mazzarella and Palumbo, 1988; Sytinsky and Oborin, 1997], and others - the anti-correlation [Sobolev et al., 1998; Shestopalov and Kharin, 2014]. Moreover, there is some skepticism in assessing the existence of such solar-terrestrial relations resulted in statistically significant seismicity variations [Love and Thomas, 2013]. It should be noted that all the mentioned conclusions (both optimistic and pessimistic) are based on correlation analysis only. Even when such analysis yields statistically significant results, the authors only assume that there is a possible triggering mechanism in the case of positive correlation, without attempts of understanding the physics of the possible relations, or provide the most general considerations. For example, it was noted [Georgieva et al., 2002] that earthquakes occur more often when the solar activity level drastically changes. During a solar flare the radiation level increases many times, which interacts with the Earth's magnetosphere resulted in its disturbance and occurrence of magnetic storm. In turn, the magnetic storms may influence to the Earth's rotation speed [Gribbin, 1971; Simpson, 1968] resulted in redistribution of stresses in the Earth crust and earthquake triggering. Some researches [Riccardi, 2007] connect the variations of seismic activity after solar flares with an increase of a number of cyclones that can act as a trigger due to sharp variations in atmospheric pressure during passage of their fronts over the earthquake source. Nevertheless, the estimations [Sobolev et al., 2012] demonstrate that such variations are substantially lower than the level of stress variations due to impact of numerous local earthquakes of moderate magnitudes (M 4-5) or due to lunar-solar tides whose influence on seismicity is still under discussion [Beeler and Lockner, 2003].

In the recent years a few papers were published where electromagnetic hypotheses were proposed on the triggering impact of geomagnetic field variations (sudden commencement storms) [Sobolev et al., 2001; Zakrzhevskaya and Sobolev, 2002; Tarasov and Tarasova, 2002], as well as diurnal Sq-variations [Duma and Ruzhin, 2003]. Particularly, results of statistical analysis obtained for different regions (Northern Tien Shan, Caucasus, Northern America, Central Europe, China, and Japan) demonstrated an evident correlation between geomagnetic field variations and a number of earthquakes both for average diurnal cycle and for a long period of about 150 years. An idea was discussed on a possible generation of additional stresses in the Earth crust due to the magnetic field variation [Duma and Ruzhin, 2003; Tren'kin, 2015]. In this paper we make an attempt to validate a possibility of earthquake triggering by solar flare based on electromagnetic triggering phenomena recently discovered in the field [Tarasov et al., 1999, 2000; Tarasov and Tarasova, 2004; Chelidze et al., 2006] and laboratory [Bogomolov et al., 2004; Avagimov et al., 2006; Lapshin et al., 2016; Chelidze et al., 2002; Novikov et al., 2017] experiments.

\section{Field and laboratory results on electric/electromagnetic triggering of earthquakes as motivation of research}

In the middle of $90^{\text {th }}$ a statistically significant impact of electrical pulses of DC current injected through emitting dipole into the earth crust on the spatial and temporal distribution of weak seismicity of Pamirs [Tarasov, 1997] and Northern Tien Shan regions [Tarasov et al., 1999, 2000; Tarasov and Tarasova, 2004; Chelidze et al., 2006] has been discovered. The grounded electrical dipole of about $4 \mathrm{~km}$ length was supplied by single pulses of electrical current of $1.5-2.5 \mathrm{kA}$, voltage of $1 \mathrm{kV}$, and duration of $2.5-10.0$ s provided by geophysical magneto-hydrodynamic (MHD) generator used for deep electromagnetic sounding of the Earth crust for monitoring the crust stress state and search of electromagnetic precursors of earthquakes. Statistically, by the epoch superposition method, with application of data of regional seismic catalogs and catalogs of firing runs of MHD generator it was found that after injection of electric current into the Earth crust the number of weak earthquakes $\left(\mathrm{M}_{\mathrm{b}}<3.0\right)$ is increased by several times with a time delay of 1-2 days, and then within 5-7 days it returns to the previous background level. A hypothesis on triggering action of electrical pulses in the stressed rocks has been proposed [Zeigarnik et al., 1999]. Laboratory experiments carried out to date with application of various press equipment and samples of geomaterials under stress-strain state demonstrated a modulation of acoustic emission by electric current pulses applied to the sample 
[Bogomolov et al., 2004; Avagimov et al., 2006; Lapshin et al., 2016]. It was shown that a response of acoustic emission to electric action characterized crack formations in the sample rises as the sample loading and maximally appears at the stress level of 0.95-0.99 of breaking stress of the geomaterial. The laboratory study of electric triggering of macro events (laboratory "earthquakes") were carried out at simplified slider system [Chelidze et al., 2002] and spring-block model [Novikov et al., 2017] simulated the seismogenic fault. The both experimental facilities are based on the idea that "stick-slip" behavior of the fault is a mechanism of earthquake preparation and occurrence [Brace and Byerlee, 1966]. In the both series of experiments the sudden slip of the movable block was initiated by injection of electrical current into a contact between the fixed and movable blocks (simulated crust fault). The triggering of slip of movable block of the spring-block model is possible only under subcritical state of the system, when the accumulated shear stresses are $0.98-0.99$ of critical value, when the sharp slip (laboratory "earthquake") occurs [Novikov et al., 2017].

Numerical calculations of DC current distribution in the crust from grounded dipole powered by MHD generator [Novikov et al., 2009] provide the current density at a depth of earthquake source locations (5-10 km) for Northern Tien Shan at a level of $10^{-7}-10^{-8} \mathrm{~A} / \mathrm{m}^{2}$. Keeping in mind that sharp variation of space weather can generate a flash of geomagnetically induced currents in the conductive subsurface structures [Lanzerotti and Gregori, 1986; Boteler et al., 1998] it was supposed that the solar flares can induce telluric currents with parameters similar to artificial DC pulsed power systems [Sorokin et al., 2012].

\section{Numerical estimations of telluric currents generated in the Earth crust by solar flares.}

For verification of idea on electromagnetic earthquake triggering by solar flares the theoretical model of electric and geomagnetic field perturbations due to changes in the ionosphere conductivity caused by solar flares [Sorokin et al., 2019] has been developed. It was assumed that the largest current amplitudes in lithosphere are induced by short-periodical oscillations of geomagnetic field [Sorokin et al., 2012]. The simple sketch of the Sun-ionospherelithosphere interaction is shown in Figure 1.

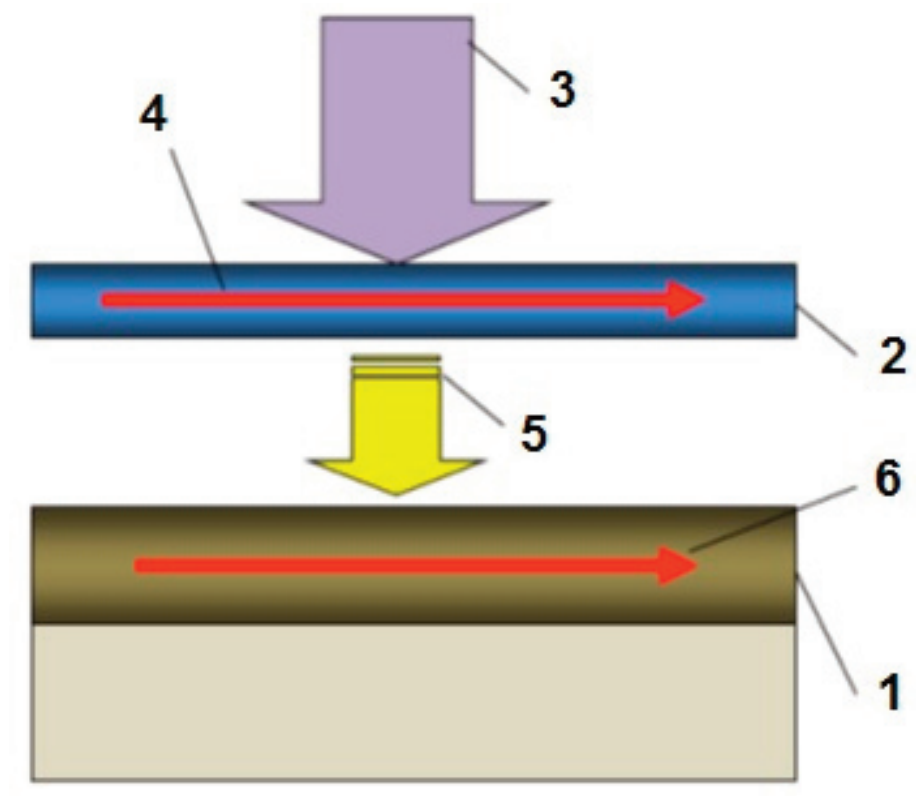

Figure 1. Diagram of the Sun-ionosphere-litosphere interaction during the solar flare. 1 - conductive layer of lithosphere; 2 - conducting layer of ionosphere; 3 - X-rays of solar flare; 4 - electric field in ionosphere; 5 - geomagnetic pulsations; 6 - induced telluric currents in conductive layer of lithosphere. 


\section{Victor Novikov et al.}

One of the mechanisms for generating geomagnetic pulsations is associated with a local change of ionospheric conductivity in the presence of an external electric field. Such a mechanism was considered in [Bell, 1976] in relation to the problems of generating Pi2 pulsations, as well as artificial oscillations of the geomagnetic field line. In these studies the processes with characteristic periods of more than 10 s were considered. The Earth-ionosphere resonator forms oscillations of the geomagnetic field with periods of 1 to 100s during ionization of ionosphere by flare radiation with a short front of its amplitude rise. Theoretical model of these processes has been developed in [Sorokin and Yaschenko, 1990] in assumption of ideally conductive Earth. For a study of parameters of induced electrical current in lithosphere the model was modified taking into account its real crust conductivity. Estimates of these parameters may be easily obtained considering the geomagnetic field as vertical. Therefore, the horizontal derivatives in equations may be neglected. It corresponds to the case when during the specific period of the electric field variation the ionosphere currents and fields diffuse in horizontal direction to the distance much less of the horizontal scale.

Numerical calculations demonstrated (see Figure 2) that as a consequence of sudden change of integral conductivities due to solar flare radiation during about ten seconds the electric fields of 0.01 to $0.1 \mathrm{mV} / \mathrm{m}$ and electric currents of density of $10^{-6}-10^{-7} \mathrm{~A} / \mathrm{m}^{2}$ are generated, that is comparable with the current density generated in the crust by artificial pulsed power sources provided earthquake triggering observed in two regions (Pamirs and Northern Tien Shan) with different geological settings. Electric current concentrates in the crust layers with increased electrical conductivity. It was shown (Figure 2) that if the electrical conductivity in the layer at a depth of $10 \mathrm{~km}$ is five times more than in the upper layer, the current density increases there by an order.

Thus, the telluric current density induced by solar flares at the depth of location of earthquake sources (5$10 \mathrm{~km}$ for Northern Tien Shan) may be similar to current density from the artificial power source affecting the regional seismic activity, that pointed to a reality of existence of solar-terrestrial electromagnetic-seismic relations and a possibility of triggering the seismic events not only by artificial power sources, but perturbations of space weather like short-period geomagnetic pulsations and strong geomagnetic storms as a result of solar flares. These results may serve as the physical background of new approach to solve the problem of short-term earthquake prediction based on electromagnetic triggering phenomena.

a)

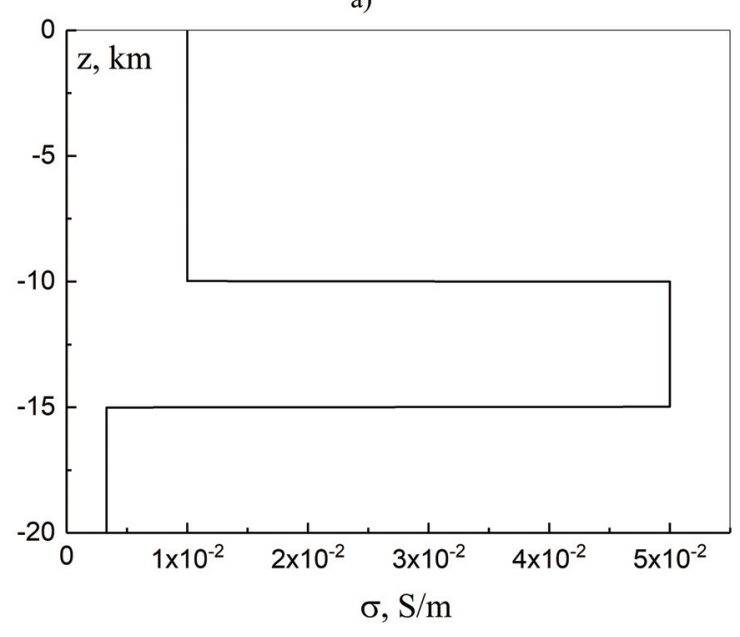

b)

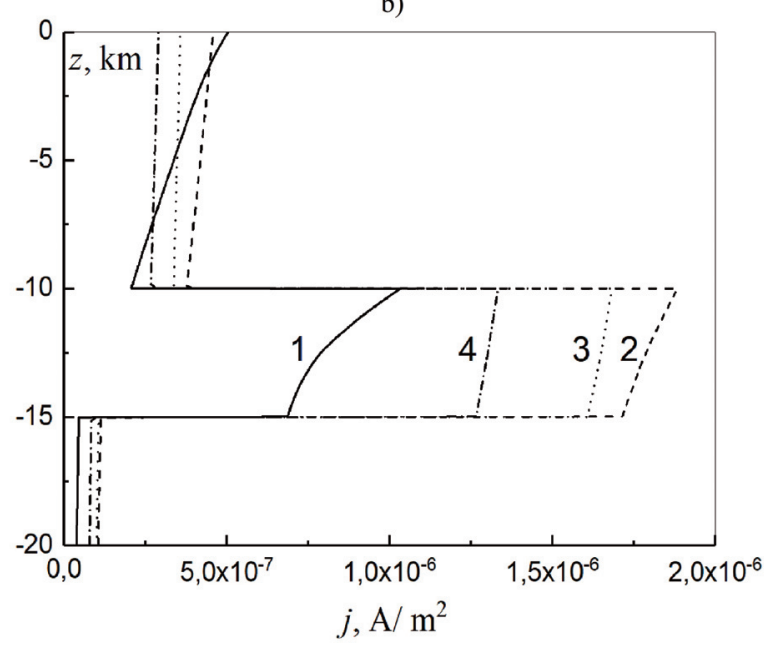

Figure 2. a) A depth dependence of electric conductivity of lithosphere used for numerical calculations; b) dependence of electric current density on the lithosphere depth at different points of time (1 - 5s, $2-10 \mathrm{~s}, 3-20 \mathrm{~s}, 4-50 \mathrm{~s})$ for characteristic duration of the front of ionosphere conductivity of $5 \mathrm{~s}$ and ionosphere conductivity of 1 [Sorokin et al., 2019]. 


\section{New evidence of earthquake triggering by solar flare}

Powerful surge of solar flash activity early in May 2017 during approaching the minimum of $24^{\text {th }}$ solar cycle was accompanied by significant disturbances of space weather. On September 6, 2017 a group of sun spots AR2673 generated a large solar flare of X9.3 class, the strongest flare over the past thirteen years. Its explosion produced a coronal mass ejection partially directed towards the Earth. We carried out a statistical analysis of the USGS and EMSC catalogs of earthquakes for determination of effect of solar flares on global seismic activity.

Considering the results published in [Sobolev et al., 2001; Zakrzhevskaya et al., 2002] indicated that the seismic response to electromagnetic impact on the lithosphere appeared during the followed 1-6 days, we choose two periods of time: one with flares of a class X (flares on September 6, 7, and 10, 2017), that is, from September 6 to 16 (blue arrow in Figure 3), and the similar background interval one day before, from August 26 to September 5 (red arrow), inclusive. It should be noted that is difficult or practically impossible at this stage of research to apply the sound statistical analysis (e.g. correlation analysis for complete data sets, both global and regional) to clear a presence of electromagnetic triggering phenomena resulted from solar flares. The earthquake faults sensitive to electromagnetic impacts should have specific parameters favorable for earthquake triggering by electromagnetic impact, namely, subcritical stress level [Novikov et al., 2017], increased electric conductivity, certain fault orientation [Han et al., 2004] for occurrence of geomagnetically induced currents of specific density required for the earthquake triggering. In many cases these parameters are unknown. In this case, we consider the simplified approach of analysis of seismicity behavior during the short period around the X class solar flares of September, 2017.
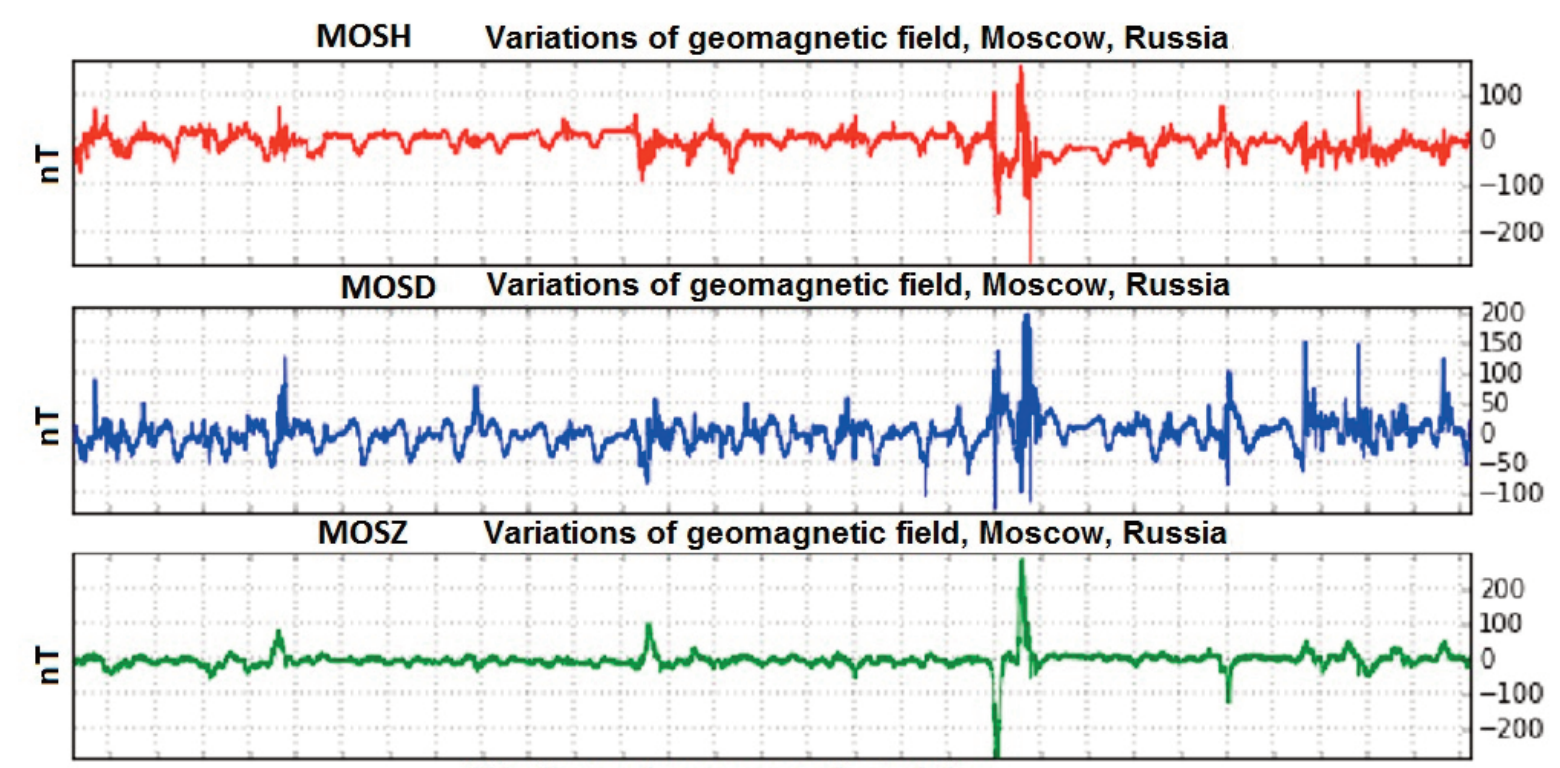

$\mathrm{K}$ indices of geomagnetic activity

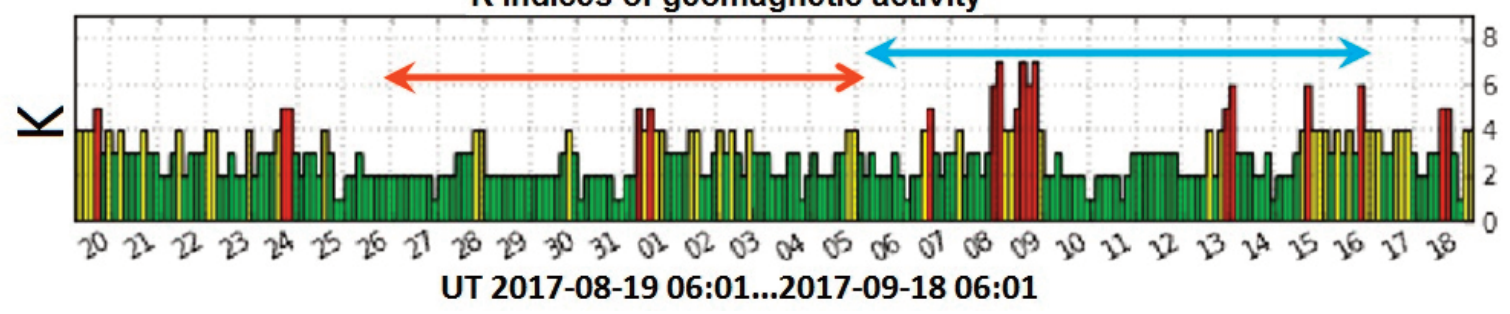

Figure 3. Magnetic activity during a month from August 20, 2017. Red and blue arrows designate periods selected for an analysis. 


\section{Victor Novikov et al.}

Figure 3 shows a magnetogram, i.e. geomagnetic field variations (three components) according to data collected in IZMIRAN observatory during a month (2017.08.20 to 2017.09.20) with the time interval of an analysis denoted by horizontal arrows. The lower part of the graph presents local index $\mathrm{K}$ of magnetic activity. Figure 4 demonstrates a rise of global seismic activity according to USGS earthquake catalog [https://earthquake.usgs.gov/earthquakes/search/] completeness of which begins from $M \geq 4.0$ according to Gutenberg-Richter law for one year preceded the solar flare of X9.3 class. A comparison of earthquake number during the same period (11 days) before and after the solar flare demonstrates a significant rise (up to $68 \%$, Figure 4) of a number of earthquakes with a magnitude of $M \geq 4.0$.

The observed pattern of earthquake activity is very similar to behavior of seismicity after injection of DC pulses into the Earth crust from artificial power systems (Figure 5). As mentioned above, we need to find the regions sensitive to electromagnetic impact. According to our study it may be the Greece region (latitude of $33.5110^{\circ}-42.4428^{\circ}$, longitude $18.8439^{\circ}-29.4344^{\circ}$ ). When we analyzed EMSC catalog [https://www.emsccsem.org/Earthquake/] with a completeness starting from $M \geq 3.0$, we found that the number of earthquakes has increased by 2.2 times ( 5 earthquakes before the solar flare, and 11 - after) in the time window of \pm 11 days. At the following stage of the research we need to concentrate on a study of the fault properties where the earthquakes occur just after the strong solar flare. Thus, based on these results we may suppose that the solar flares can provoke the earthquake occurrence. One of the possible reasons of earthquake triggering is the sharp increase of telluric currents induced in the conductive areas of geological faults matured for earthquake occurrence [Han et al., 2004].

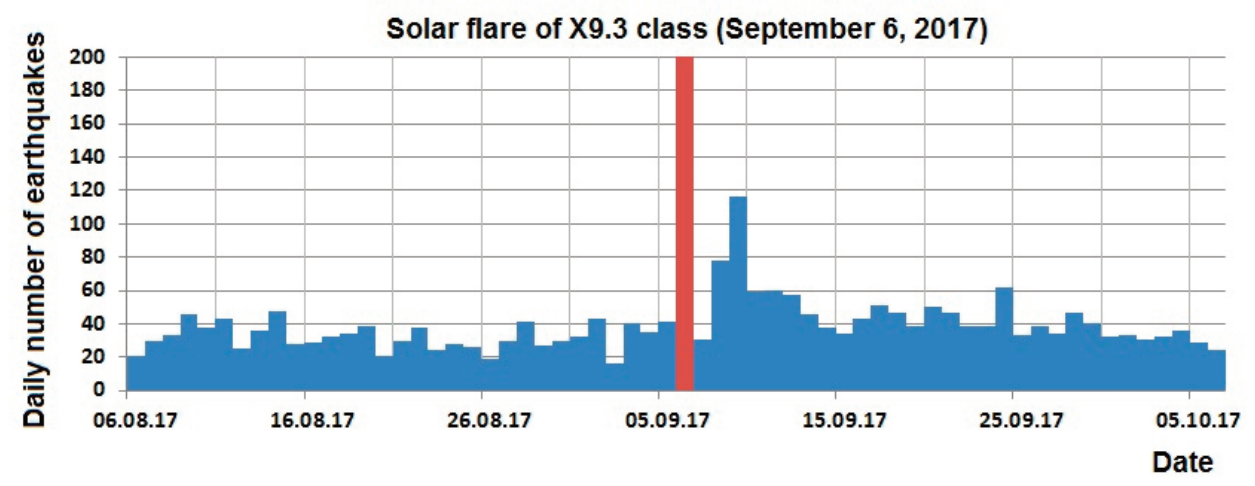

Figure 4. Global daily number of earthquakes before and after the solar flare of X9.3 class (vertical red line) of September 6, 2017 (11:53 UTC). Catalog USGS, M $\geq 4.0$ (2016-2017, average annual daily number of earthquakes is 38.37).

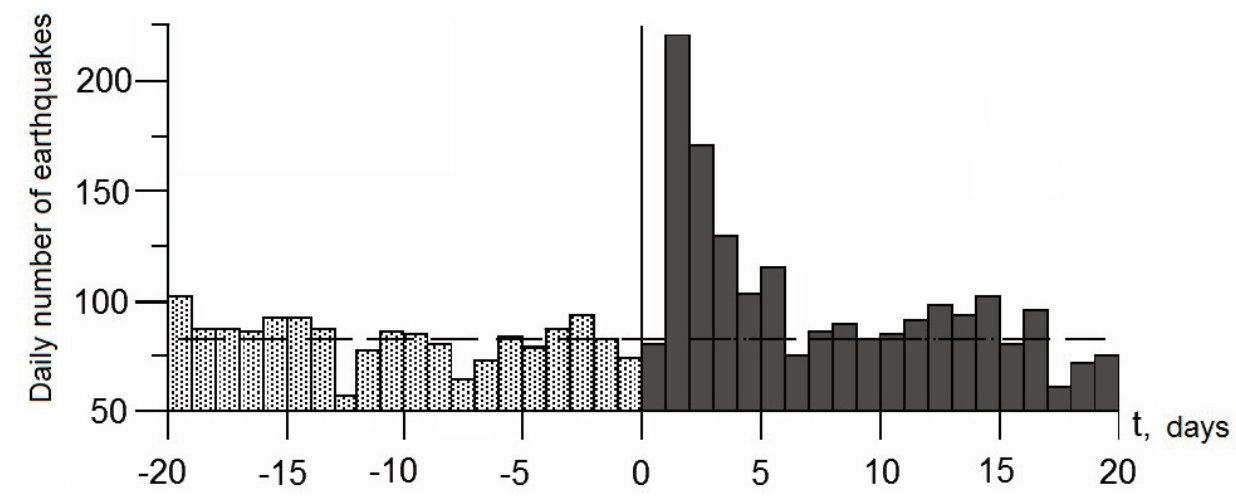

Figure 5. Daily number of earthquakes in the upper layer $(0-5 \mathrm{~km})$ of the Earth crust of Bishkek geodynamic al proving ground (Northern Tien Shan) before $(t<0)$ and after $(t>0)$ operation of MHD generator for DC current injection into the Earth crust [Tarasov and Tarasova, 2004]. 


\section{Conclusion}

The overview of studies completed to-date on a relation of the Earth's seismicity and solar processes demonstrates the fuzzy and contradictory results. The main problem of this research is a lack of physical explanation of a mechanism of earthquake triggering by strong variations of space weather conditions. Based on results obtained in the field and laboratory experiments on earthquake triggering by DC pulses injection into the Earth crust we supposed that the similar triggering phenomena may occur after the strong electromagnetic impact to the earthquake source due to solar flares or geomagnetic storms. This idea is supported by results of numerical estimations made with an application of developed physical model of possible mechanism of stimulation of seismic activity by ionizing radiation of solar flares. It was shown that telluric currents induced by geomagnetic pulsations generated by solar flare have the similar density at the depth of earthquake source location $\left(10^{-6} \mathrm{~A} / \mathrm{m}^{2}\right)$ in comparison with the current density generated by artificial power sources $\left(10^{-7}-10^{-8} \mathrm{~A} / \mathrm{m}^{2}\right)$ resulted in observed redistribution of seismic activity in the regions of Pamirs and Northern Tien Shan. These results are supported by the simple statistical analysis of global and regional (Greece) seismicity behavior during the solar flare of X9.3 class occurred on September 6, 2017 (the strongest flare over the past thirteen years). A number of earthquakes after the strong solar flare increased both for global and regional scales by 68 and $120 \%$, correspondingly. The global number of earthquakes (USGS catalog, $M \geq 4$ ) for time window of \pm 10 days after the solar flare has increased by $68 \%$, and the regional seismicity (Greece, EMSC catalog, $M \geq 3$ ) has increased by $120 \%$.

Based on the results described above we can suppose that the earthquake triggering due to impact of strong solar flares is possible. The physical mechanism of interaction of week electrical current with rocks under critical stress-strain state is not clear today. The effect of seismicity response to electrical action may be explained either by electric current concentration in the narrow highly conductive fault zones [Han et al., 2004] or by secondary triggering mechanisms driven by electric pulses, like electromagnetic stimulation of fluid migration into the fault zone resulted in reduction of the fault strength properties [Novikov and Novikova, 2014]. It should be noted that these hypotheses have only a phenomenological character without a detailed theoretical justification and experimental verification. New fundamental knowledge is needed on the mechanisms of interaction of the electromagnetic field with rocks, as well as on the influence of electric/electromagnetic fields in combination with natural geophysical fields on deformation processes in the fault with specific parameters (electrical conductivity, orientation and maturity for earthquake occurrence).

Acknowledgements. The work was supported by RFBR grant No. 18-05-00962. We thank for constructive comments of Paolo Harabaglia and an anonymous referee, which improved the manuscript.

\section{References}

Avagimov, A. A., V. A. Zeigarnik and V. N. Klyuchkin (2006). On the structure of acoustic emission of model samples in response to an external energy action, Phys Solid Earth, 42: 824-829.

Beeler, N. M. and D. A. Lockner (2003). Why earthquakes correlate weakly with the solid Earth tides: Effects of periodic stress on the rate and probability of earthquake occurrence, J. Geophys. Res., 108(B8), 2391.

Bell, T.F. (1976). ULF wave generation through particle precipitation induced by VLF transmitters, J Geophys. Res., $81: 3316$.

Bogomolov, L.M., P. V. Il'ichev, V. A. Novikov, V.I. Okunev, V.N. Sychev and A.S. Zakupin (2004). Acoustic emissions response of rocks to electric power action as seismic-electric effect manifestation, Ann. Geophys., 47, 1, 6572 .

Boteler, D. H., R. J. Pirjola and H. Nevanlinna (1998). The effects of geomagnetic disturbances on electrical systems at the Earth's surface, Adv. Space Res., 22: 17-27.

Brace, W.F. and J. D. Byerlee (1966). Stick-slip as a mechanism for earthquakes, Science, 153: 990-992.

Chelidze, T., N. Varamashvili, M. Devidze, Z. Chelidze, V. Chikladze and T. Matcharashvili (2002). Laboratory study of electromagnetic initiation of slip, Ann. Geophys., 45, 5, 587-598.

Chelidze, T., V. De Rubeis, T. Matcharshvili and P. Tosi (2006). Influence of strong electromagnetic discharges on the 


\section{Victor Novikov et al.}

dynamic of earthquakes time distribution in the Bishkek test area (Central Asia), Ann. Geophys., 49, 4-5, 961975.

Duma, G. and Yu. Ruzhin (2003). Diurnal changes of earthquake activity and geomagnetic Sq variations, Nat Hazards Earth Sys., 3-4: 171-177.

EMSC earthquake catalogue - https://www.emsc-csem.org/Earthquake/

Georgieva, K., B. Kirov and D. Atanasov (2002). On the relation between solar activity and seismicity on different time scales, J. Atmosph. Electr., 22: 291-300.

Gomberg, J., N. M. Beeler and M. L. Blanpied (1998). Earthquake triggering by static and dynamic deformations, J. Geophys. Res. 103: 24411-24426.

Gribbin, J. (1971). Relation of sunspot and earthquake activity, Science, 173: 558.

Han, Y., Z. Guo, J. Wu and L. Ma (2004). Possible triggering of solar activity to big earthquakes (Ms $\geqslant 8$ ) in faults with near west-east strike in China, Sci. China Ser. G: Phy \& Ast, 47: 173-181.

Lanzerotti, L. J. and G. P. Gregori (1986). Telluric currents: The natural environment and interaction with man-made systems, in: The Earth's Electrical Environment, The National Academic Press, Washington, D.C.: 232-257.

Lapshin, V. B., A. V. Patonin, A. V. Ponomarev, M. G. Potanina, V. B. Smirnov and S. M. Stroganova (2016). Initiation of Acoustic Emission in Fluid-Saturated Sandstone Samples, Doklady Earth Sciences, 469: 705-709.

Love, J. J. and J. N. Thomas (2013). Insignificant solar-terrestrial triggering of earthquakes. Geophys. Res. Lett., 40: $1165-1170$.

Mazzarella, A. and A. Palumbo (1988). Solar, geomagnetic and seismic activity, Il Nuovo Cimento, C 11: 353-364.

Novikov, V. A., V. I. Okunev, V. N. Klyuchkin, J. Liu, Yu. Ya. Ruzhin and X. Shen (2017). Electrical triggering of earthquakes: Results of laboratory experiments at spring-block models, Earthq. Sci., 30: 167-172.

Novikov, V.A. and E. O. Novikova (2014). Electromagnetic stimulation of fluid migration into fault area and earthquake triggering phenomena, Geophys. Res. Abstr., 16: EGU2014-12790.

Novikov, V., V. Zeigarnik, Yu. Konev and V. Rickman (2009). Electric Triggering of Crack Formations in the Fault for Release of Tectonic Stresses, Eos Trans. AGU, 90(52), Fall Meet. Suppl., Abstract S51C-1447.

Riccardi, U., J. Hinderer and J. P. Boy (2007). On the efficiency of barometric arrays to improve the corrections of atmospheric effects on gravity data, Phys. Earth Planet. Int., 161: 224-242.

Shestopalov, I. P. and E. P. Kharin (2014). Relationship between solar activity and global seismicity and neutrons of terrestrial origin, Russ. J. Earth. Sci., 14: ES1002.

Simpson, I.F. (1968). Solar activity as a triggering mechanism for earthquakes, Earth and Planet Sci. Letter., 3: 417425.

Sobolev, G. A., I. P. Shestopalov and E. P. Kharin (1998). Implications of Solar Flares for the Seismic Activity of the Earth, Izv., Phys. Solid Earth, 34: 603-607.

Sobolev, G. A., N. A. Zakrzhevskaya and D. G. Sobolev (2012). The Effects of Cyclones on seismicity, J. Volcanol. Seismol., 6: 89-99.

Sobolev, G.A., N. A. Zakrzhevskaya and E. P. Kharin (2001). On the coupling of seismicity with magnetic storms, Izv., Phys. Solid Earth, 11:62-72.

Sorokin, V. M. and A. K. Yashchenko (1990). Generation of short-period oscillations of geomagnetic field as a result of large-scale disturbances of ionosphere conductivity, Geomagn. Aeron, 30: 425-428.

Sorokin, V. M., A. K. Yaschenko, Yu. Ya. Ruzhin and V.A. Novikov (2012). Model for solar flare influence to the seismic activity, in: Proc. EMSEV-2012 Workshop. Gotemba, Japan. October 1-4, 2012. Abstr. 3-10p.

Sorokin, V. M., A. K. Yaschenko and V.A. Novikov (2019). A possible mechanism of stimulation of seismic activity by ionizing radiation of solar flares, Earthq Sci, 32: 26-34.

Sytinsky, A. D. and D. A. Oborin (1997). Influence of perturbations of interplanetary medium on seismicity and atmosphere of the Earth, Geomagn. Aeron., 37: 138-141.

Tarasov, N. T. and N. V. Tarasova (2002). The effect of geomagnetic storms on the seismicity, in: Proc. of the $3^{\text {rd }}$ International Workshop on Magnetic, Electric and Electromagnetic Methods in Seismology and Volcanology, Moscow, Russia, 3-6 September 2002: 206-208.

Tarasov, N. T., N. V. Tarasova, V. A. Zeigarnik and A. A. Avagimov (2000). The effect of high energy electromagnetic pulses on seismicity in Central Asia and Kazakhstan, J. Volcanol. Seismol., 21: 627-639.

Tarasov, N. T. and N. V. Tarasova (2004). Spatial-temporal structure of seismicity of the North Tien Shan and its change under effect of high energy electromagnetic pulses, Ann. Geoph., 47: 199-212. 
Tren'kin, A. A. (2015). Possible influence of telluric current on seismicity of the Earth's crust in seismoactive zones, Geomagn. Aeron., 55: 133-138.

USGS earthquake catalogue - https://earthquake.usgs.gov/earthquakes/search/

Wolf, R. (1853). On the periodic return of the minimum of sun-spots: The agreement between those periods and the variations of magnetic declination, Philos. Magazine, 5: 67.

Zakrzhevskaya, N. A. and G. A. Sobolev (2002). On the seismicity effect of magnetic storms, Izv., Phys. Solid Earth, 38: $249-261$

Zeigarnik, V. A., A. A. Avagimov and N. T. Tarasov (1999). Managing earthquakes? Science in Russia, 2: 16-21. 